\title{
Shuttle walking test como instrumento de avaliação da capacidade funcional: uma revisão da literatura
}

\author{
Shuttle walking test as an instrument for assessment of functional \\ capacity: a literature review
}

\begin{abstract}
Débora Pantuso Monteiro ${ }^{1}$, Raquel Rodrigues Britto ${ }^{2}$, Maria Luiza Vieira Carvalho ${ }^{3}$, Dayane Montemezzo ${ }^{4}$, Verônica Franco Parreira ${ }^{5}$, Danielle Aparecida Gomes Pereira ${ }^{6}$
\end{abstract}

\section{RESUMO}

Introdução: O Shuttle walking test (SWT) foi originalmente desenvolvido para indivíduos com doença pulmonar obstrutiva crônica (DPOC). Sua aplicação na avaliação funcional em outras condições de saúde vem sendo empregada. Objetivo: Fazer uma revisão de literatura sobre o uso do Shuttle walking test na avaliação da capacidade funcional. Materiais e Métodos: A revisão da literatura foi realizada nas bases de dados Medline, Lilacs e Scielo considerando a combinação do descritor principal Shuttle walking test com os descritores em português e inglês: validade, reprodutibilidade, confiabilidade, sensibilidade, diferença mínima clinicamente significativa e prognóstico.

Resultados: Foram selecionados 37 artigos que apresentavam o SWT como desfecho cardiorrespiratório de interesse nas seguintes condições de saúde: insuficiência cardíaca, doença arterial coronariana, DPOC, transplante cardíaco, doença arterial obstrutiva periférica, fibrose cística, fibrose pulmonar idiopática e pacientes com marcapasso.

Conclusão: O SWT é um teste de caminhada válido, confiável e seguro para a avaliação da capacidade funcional em diversas condições de saúde. É útil na prática clínica para avaliar respostas a intervenções e prognóstico.

Palavras-chave: teste de esforço; condicionamento físico humano; fisioterapia.

\begin{abstract}
Introduction: The Shuttle walking test (SWT) was originally developed for individuals with chronic obstructive pulmonary disease (COPD). It is also been used to evaluate the functional capacity in other health conditions.

Objective: To do a review about the use of the Shuttle walking test to assess the functional capacity.

Materials and Methods: A literature review was performed in Medline, Lilacs and Scielo considering the keywords: Shuttle walking test, validity, reproducibility, reliability, sensibility, minimum clinically significance difference and prognostic.

Results: Thirty seven articles mentioned the SWT as a variable of interest. Besides COPD it was used to evaluate patients with heart failure, coronary arterial disease, heart transplantation, peripheral arterial obstructive disease, cystic fibrosis, idiopathic pulmonary fibrosis and patients with pacemakers.

Conclusions: The SWT is valid, reliable and safe to assess cardiorespiratory fitness and functional capacity in different health conditions. It is usable on clinical practice to evaluate responses to treatments and also for prognostic.
\end{abstract}

Keywords: exercise test; human physical conditioning; physical therapy specialty.

\footnotetext{
${ }^{1}$ Fisioterapeuta. Mestre em Ciências da Reabilitação pela Universidade Federal de Minas Gerais (UFMG), Belo Horizonte-MG, Brasil. ${ }^{2}$ Fisioterapeuta. Doutora em Ciência Biológicas. Professora Associada do Departamento de Fisioterapia da Escola de Educação Física, Fisioterapia e Terapia Ocupacional da UFMG.

${ }^{3}$ Fisioterapeuta. Mestre em Ciências da Reabilitação pela UFMG.

${ }^{4}$ Fisioterapeuta. Doutoranda do Programa de Pós Graduação em Ciências da Reabilitação (UFMG).

${ }^{5}$ Fisioterapeuta. Doutora em Fisioterapia e Reabilitação. Professora Associada do Departamento de Fisioterapia da Escola de Educação Física, Fisioterapia e Terapia Ocupacional da UFMG.

${ }^{6}$ Fisioterapeuta. Doutora em Ciências da Reabilitação. Professora Adjunta do Departamento de Fisioterapia da Escola de Educação Física, Fisioterapia e Terapia Ocupacional da UFMG.
} 


\section{INTRODUÇÃO}

O teste de esforço cardiopulmonar (TECP) máximo é considerado padrão-ouro na avaliação da capacidade física, mas sua aplicação torna-se limitada naqueles indivíduos com capacidade funcional restrita ou quando o esforço máximo é contra-indicado ${ }^{1}$. Além disso, o TECP requer equipamentos específicos para monitorização e equipe especializada. Considerando as particularidades do TECP, uma opção para a avaliação da capacidade física são os testes que exijam menor nível de esforço, como os submáximos. Esses testes podem ter maior aplicabilidade clínica na assistência às pessoas com limitações no desempenho de atividades físicas, na avaliação dos resultados de diferentes intervenções, da estabilidade clínica e do prognóstico ${ }^{2-6}$.

Dentre os testes submáximos, os testes de caminhada têm sido utilizados na prática clínica desde a década de 1960, quando Kenneth Cooper ${ }^{7}$ desenvolveu o teste de caminhada de 12 minutos, com a finalidade de avaliar a capacidade funcional e predizer o consumo máximo de oxigênio $\left(\mathrm{VO}_{2} \mathrm{max}\right)$ de indivíduos saudáveis. Atualmente, esse teste foi substituído pelo teste de caminhada de seis minutos (TC6) uma vez que há forte correlação entre $\mathrm{o} \quad \mathrm{VO}_{2} \max$ medido em ambos $(r=0,955)^{8}$. Apesar de amplamente usado, o TC6 tem algumas limitações, como a motivação do indivíduo, o encorajamento fornecido pelo avaliador ${ }^{9}$ e a velocidade auto selecionada durante 0 teste ${ }^{8-10}$, que podem influenciar na distância percorrida.

Diante disso, Singh et al. ${ }^{11}$, em 1992, desenvolveram o Shuttle walking test (SWT), referido em artigo brasileiro como Teste de Deslocamento Bidirecional Progressivo $^{12}$. Trata-se de um teste simples, incremental, com velocidade controlada por sinais sonoros 0 qual tem como finalidade avaliar o desempenho do indivíduo levando em consideração os sintomas limitantes. O SWT foi criado como um instrumento de avaliação para indivíduos com doença pulmonar obstrutiva crônica (DPOC) ${ }^{11}$; porém, tem sido utilizado também em outras condições de saúde ${ }^{13-27}$.

Com a popularização do SWT na avaliação de outras condições de saúde além da DPOC, é importante conhecer sua aplicabilidade e os resultados encontrados na avaliação funcional de outras populações. Diante do exposto, o objetivo deste estudo é fazer uma revisão de literatura sobre o uso do Shuttle walking test na avaliação da capacidade funcional.

\section{MATERIAIS E MÉTODOS}

A revisão da literatura foi realizada nas bases de dados Medline, Lilacs e Scielo considerando a combinação do descritor principal Shuttle walking test com os descritores nos idiomas português/inglês: validade/validity, reprodutibilidade/reliability, confiabilidade/reliability, sensibilidade/sensibility, diferença mínima clinicamente significativa/minimal clinically important difference e prognóstico/prognostic. Não foi estabelecido um período limite de tempo da publicação dos estudos sendo incluídos todos os artigos que apresentaram o SWT como desfecho cardiorrespiratório até agosto de 2013, independente do tipo do estudo. Artigos publicados nos idiomas inglês, português e espanhol poderiam ser incluídos. Além disto, foi realizada busca manual nas referências dos artigos encontrados.

\section{RESULTADOS E DISCUSSÃO}

A partir da leitura do título foram encontrados 652 artigos. Desses 593 foram excluídos por não apresentarem o SWT como desfecho principal e 59 por

TABELA 1 - Condições de saúde avaliadas nos 37 estudos identificados.

\begin{tabular}{lcc}
\hline $\begin{array}{l}\text { Condição de saúde } \\
\text { estudada }\end{array}$ & $\%$ & Referência \\
\hline $\begin{array}{l}\text { Doença pulmonar } \\
\text { obstrutiva crônica }\end{array}$ & 37,84 & $\begin{array}{c}3,5,6,11,13,17,18,19, \\
20,25,30,31,34,37\end{array}$ \\
$\begin{array}{l}\text { Doença } \\
\text { cardiovascular }\end{array}$ & 10,81 & $33,38,39,40$ \\
$\begin{array}{l}\text { Insuficiência cardíaca } \\
\text { Fibrose cística }\end{array}$ & 10,81 & $2,21,32,36$ \\
$\begin{array}{l}\text { Doença arterial } \\
\text { obstrutiva periférica }\end{array}$ & 8,11 & $14,15,16$ \\
$\begin{array}{l}\text { Transplante cardíaco } \\
\text { Fibrose pulmonar }\end{array}$ & 2,7 & 12,22 \\
$\begin{array}{l}\text { Pacientes com } \\
\text { marcapasso }\end{array}$ & 2,7 & 4 \\
$\begin{array}{l}\text { Doenças } \\
\text { cardiorespiratórias }\end{array}$ & 2,7 & 23 \\
$\begin{array}{l}\text { Pós operatório de } \\
\text { câncer de pulmão }\end{array}$ & 2,7 & 27 \\
\hline
\end{tabular}


duplicidade. Assim, 36 artigos foram selecionados a partir da busca nas bases de dados e um por busca manual, totalizando 37 artigos na presente revisão (tabela 1).

\section{Descrição do SWT}

O SWT foi criado a partir do Shuttle run test ${ }^{28}$. Inicialmente apresentava 10 estágios, sendo que após os primeiros resultados, foi desenvolvido um protocolo modificado com 12 estágios, que inicia com velocidade mais lenta permitindo aos indivíduos mais debilitados melhor adaptação ${ }^{11}$.

O SWT apresenta 12 estágios com um minuto de duração cada, velocidade inicial de 0,5 metros/segundo $(\mathrm{m} / \mathrm{s})$, sendo a cada minuto acrescentado $0,17 \mathrm{~m} / \mathrm{s}$ (equivalente a 10 metros/minuto), conforme descrito na tabela 2. O avaliador pode fornecer comando verbal padronizado ao fim de cada estágio com intuito de informar ao indivíduo sobre o aumento da velocidade de caminhada $^{11}$. A velocidade de caminhada é determinada por meio de dois tipos diferentes de sinais sonoros: (1) um sinal (bipe) único que indica mudança de direção e (2) um sinal (bipe) triplo que indica mudança de direção e de estágio.

TABELA 2 - Protocolo do Shuttle walking test.

\begin{tabular}{lcc}
\hline Estágio & Velocidade $(\mathbf{m} / \mathbf{s e g})$ & $\mathbf{N}^{\circ}$ de Percursos \\
\hline 1 & 0,5 & 3 \\
2 & 0,67 & 4 \\
3 & 0,84 & 5 \\
4 & 1,01 & 6 \\
5 & 1,18 & 7 \\
6 & 1,35 & 8 \\
7 & 1,52 & 9 \\
8 & 1,69 & 10 \\
9 & 1,86 & 11 \\
10 & 2,03 & 12 \\
11 & 2,20 & 13 \\
12 & 2,37 & 14 \\
\hline
\end{tabular}

Modificado de Singh et al.11, 1992.

\section{Procedimento de aplicação}

Para realização do SWT utiliza-se uma pista de 10 metros, demarcada por dois cones, com distância de nove metros entre eles e meio metro além de cada cone para o retorno (figura 1). O indivíduo é instruído a caminhar de um cone ao outro, de acordo com o ritmo determinado pelos sinais sonoros, até a fadiga ou presença de sintoma limitante. Um dos critérios de interrupção do teste é a incapacidade de manter o ritmo de deslocamento, ou seja, quando o indivíduo não alcança o cone subsequente, por duas vezes consecutivas, dentro do tempo estabelecido pelos sinais sonoros $^{12}$. Além disso, o teste deverá ser interrompido caso o indivíduo apresente valores de frequência cardíaca superiores a $85 \%$ da máxima prevista ou queda da saturação ${ }^{11}$.

Os seguintes equipamentos são utilizados para aplicação do SWT: cronômetro, cones para demarcação da pista, aparelho de som e CD contendo a gravação do SWT, esfigmomanômetro e estetoscópio, cardiofrequencímetro, oxímetro de pulso e escala de percepção subjetiva de esforço.

O teste deverá ser realizado pelo menos duas horas após as refeições. Os sujeitos devem utilizar roupas e calçados confortáveis e manter a medicação usual ${ }^{29}$. Antes da realização do teste serão aferidas pressão arterial, frequência cardíaca, saturação periférica de oxigênio e frequência respiratória ${ }^{24}$. Além disso, a existência de possíveis contra-indicações deve ser avaliada $^{29}$.

Durante a realização do SWT, o avaliador poderá caminhar ao lado do indivíduo no primeiro estágio com objetivo de melhor compreensão da dinâmica do teste ${ }^{11}$ ou nos casos em que a oxigenoterapia seja necessária. A frequência cardíaca ao final de cada estágio deverá ser registrada. É importante destacar que os parâmetros clínicos específicos para determinadas condições de saúde devem ser igualmente observados. Ao término do teste, os dados vitais e a percepção subjetiva de esforço devem ser analisados.

Em relação às variáveis fornecidas pelo SWT devem ser registrados distância máxima percorrida, velocidade máxima alcançada, pressão arterial, frequência 


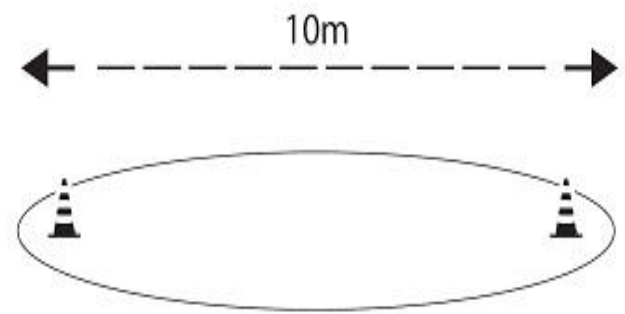

FIGURA 1 - Esquema de organização da dinâmica do Shuttle walking test. Modificado de Singh et al. ${ }^{11}, 1992$.

cardíaca, percepção subjetiva de esforço, estágio e respectivo percurso no qual o teste foi interrompido ${ }^{11}$.

\section{Utilização do SWT na clínica}

O SWT pode ser amplamente aplicado a indivíduos com diferentes condições de saúde e faixas etárias, bem como, com diferentes finalidades ${ }^{13-27}$, sendo as mais frequentes: avaliação de resposta às intervenções $^{30}$; avaliação da capacidade funcional ${ }^{2,31}$; avaliação de prognóstico ${ }^{2,32}$.

O SWT é um instrumento com baixo índice de complicações durante sua execução, embora poucos estudos tenham avaliado sua segurança ${ }^{2,33}$. No estudo de Pulz et al. ${ }^{2}$, indivíduos com insuficiência cardíaca foram monitorados com eletrocardiograma durante a realização do SWT e foi verificado que é um teste seguro. De acordo com o American College of Sports Medicine (ACSM) é recomendada a presença de profissionais treinados, o conhecimento sobre as técnicas de ressuscitação e a realização do teste em local de fácil acesso aos serviços de emergência ${ }^{29}$.

O SWT é um instrumento considerado válido ${ }^{2-6,11,34,35}$ e confiável ${ }^{4,11,12,33,36-40}$ para avaliação da capacidade funcional. Os resultados do estudo de Singh et al. ${ }^{34}$ mostraram forte correlação $(r=0,88)$ entre o desempenho no SWT e o $\mathrm{VO}_{2} \mathrm{max}$ de indivíduos com DPOC. Para indivíduos com insuficiência cardíaca também foi encontrada forte associação ( $r=0,79$, $\mathrm{p}<0,001$ ) entre $\circ \mathrm{VO}_{2}$ pico e a distância percorrida no $\mathrm{SWT}^{2}$. Em indivíduos a espera de um transplante cardíaco, Lewis et al. $^{4}$ encontraram moderada associação $(r=0,73, p=0,0001)$ entre essas variáveis.
A confiabilidade do SWT foi comparada a do TC6 em indivíduos com claudicação intermitente devido a doença arterial obstrutiva periférica no estudo de $\mathrm{Da}$ Cunha-Filho et al. ${ }^{12}$. Não houve diferença estatisticamente significativa entre a distância percorrida nos dois SWT realizados, o que confirma a reprodutibilidade do teste para essa população dispensando a realização de um teste para familiarização (ICC=0,95, $p=0,0005)$. Adicionalmente, quando comparado ao TC6, o SWT apresentou coeficientes de confiabilidade maiores. Pepera et al. ${ }^{38}$, avaliaram a confiabilidade teste-reteste em longo prazo do SWT, em indivíduo na fase IV de reabilitação cardíaca. Foram realizados dois SWT com intervalo mínimo de oito semanas e não houve diferença estatisticamente significativa entre a distância percorrida (ICC=0,80, 95\% IC 0,62 a 0,90), não sendo necessária a realização de familiarização.

Uma forma de interpretação dos resultados do SWT é por meio da comparação das variáveis obtidas no teste pré e pós-intervenção. Dentre essas, tem-se a distância percorrida na qual o acréscimo de 47,5 metros é considerado como um aumento clinicamente relevante, ou seja, a obtenção de melhora nos parâmetros clínicos e na percepção de pacientes com DPOC ${ }^{30}$. Não foram encontrados estudos que avaliassem a responsividade do SWT em outras populações. Porém, o SWT demonstrou ser bom preditivo de prognóstico em indivíduos com Insuficiência Cardíaca (IC), inclusive para aqueles aguardando transplante cardíaco. Uma distância percorrida no teste acima de 450 metros é um discriminador sensível dos indivíduos com $\mathrm{VO}_{2} \max$ acima de $14 \mathrm{~mL} / \mathrm{Kg} \cdot \mathrm{min}^{-1}$, utilizado como critério indicativo de transplante cardíaco ${ }^{4}$. Ainda em indivíduos com IC, o SWT mostrou-se melhor preditivo de eventos cardíacos em um ano, quando comparado com o TC6. Nesse sentido, indivíduos que percorreram distâncias menores que 450 metros apresentaram maior risco de eventos cardíacos ${ }^{32}$. A medida direta do $\mathrm{VO}_{2}$ pico ainda é superior ao SWT em predizer a sobrevivência nessa população, pelo menos naqueles com capacidade funcional preservada ${ }^{2}$.

Até o momento foram identificados três estudos ${ }^{41-43}$ que propuseram equações de referência para o SWT 
TABELA 3 - Equações preditivas disponíveis na literatura pesquisada.

\begin{tabular}{|c|c|c|c|c|}
\hline Autor & População & Equação & EPE & $\mathbf{R}^{2}$ \\
\hline $\begin{array}{l}\text { Jürgensen et al. }{ }^{41} \\
2011\end{array}$ & $\begin{array}{c}40 \text { a } 84 \text { anos } \\
n=131\end{array}$ & $\begin{aligned} \text { SWT prev }= & 374,004-(6,782 * \text { id }(\text { anos }))-\left(2,328{ }^{*} \text { peso }(\mathrm{kg})\right)+ \\
& \left(3,865{ }^{*} \text { altura }(\mathrm{cm})\right)+\left(115,937^{*} \text { sexo }^{\#}\right)\end{aligned}$ & $\begin{array}{l}114 \\
732\end{array}$ & 0,503 \\
\hline $\begin{array}{l}\text { Dourado et al. }{ }^{42} \\
2011\end{array}$ & $\begin{array}{l}\text { Acima de } 40 \text { anos } \\
n=98\end{array}$ & $\begin{aligned} \text { SWT prev }= & 34,608-(4,384 \text { * id (anos }))-(2,949 * \text { peso }(\mathrm{kg}))+ \\
& (553,336 \text { * altura }(\mathrm{m}))+\left(114,387^{*} \text { sexo }^{\#}\right)\end{aligned}$ & 79,4 & 0,649 \\
\hline $\begin{array}{c}\text { Dourado et al }{ }^{43} \\
2013\end{array}$ & $\begin{array}{l}\text { Acima de } 40 \text { anos } \\
n=103\end{array}$ & $\begin{array}{c}\text { SWT prev }=347,7-(7,2 \text { * id }(\text { anos }))-\left(3^{*} \text { peso }(\mathrm{kg})\right)+\left(472,3^{*}\right. \\
\text { altura }(\mathrm{m}))+\left(137,2{ }^{*} \text { sexo }\right)\end{array}$ & 97,8 & 0,65 \\
\hline
\end{tabular}

cm: centímetros; EPE: erro padrão da estimativa; id: idade; IMC: índice de massa corporal; m: metros; n: número amostral; SWT prev: distância prevista pela fórmula (em metros); $\mathrm{R}^{2}$ : coeficiente de determinação. \#Atribuir 1 para sexo masculino e 0 para o sexo feminino.

(tabela 3). Foi identificado um quarto estudo, realizado por Probst et al. ${ }^{44}$, cujos participantes foram indivíduos saudáveis e na aplicação do protocolo do teste foi permitido correr. Sendo assim, recomenda-se que os resultados deste estudo sejam aplicados com cautela em populações distintas daquela na qual o estudo foi realizado.

Sumarizando, o SWT é um teste de caminhada válido, confiável e seguro para a avaliação da capacidade funcional em diversas condições de saúde, em especial em doenças respiratórias, cardíacas e vasculares. É útil na prática clínica para avaliar respostas a intervenções e prognóstico. Além disso, estão disponíveis equações de referência em estudos realizados em população brasileira, que facilita a aplicabilidade clínica do teste no Brasil. No entanto, ainda são poucos os estudos que avaliaram a responsividade do SWT. Além disso, o SWT é considerado um teste simples, bem tolerado, de baixo custo e requer poucos equipamentos para sua aplicação. Portanto, apresenta características satisfatórias para ser utilizado com maior frequência na prática clínica.

\section{REFERÊNCIAS}

1. Noonan V, Dean E. Submaximal exercise testing: clinical application and interpretation. Phys Ther. 2000; 80(8):782807.

2. Pulz C, Diniz RV, Alves AN, Tebexreni AS, Carvalho AC, de Paola AA, Almeida DR. Incremental shuttle and sixminute walking tests in the assessment of functional capacity in chronic heart failure. Can J Cardiol. 2008; 24(2):131-5.

3. Arnardottir $\mathrm{RH}$, Emtner $\mathrm{M}$, Hedenstrom $\mathrm{H}$, Larsson $\mathrm{K}$, Boman G. Peak exercise capacity estimated from incremental shuttle walking test in patients with COPD: a methodological study. Respir Res. 2006; 7:127.

4. Lewis ME, Newall C, Townend JN, Hill SL, Bonser RS. Incremental shuttle walk test in the assessment of patients for heart transplantation. Heart. 2001; 86(2):183-7.
5. Onorati $P$, Antonucci R, Valli G, Berton E, De MF, Serra $P$, Palange $P$. Non-invasive evaluation of gas exchange during a shuttle walking test vs. a 6-min walking test to assess exercise tolerance in COPD patients. Eur $\mathrm{J} \mathrm{Appl}$ Physiol. 2003; 89(3-4):331-6.

6. Turner SE, Eastwood PR, Cecins NM, Hillman DR, Jenkins SC. Physiologic responses to incremental and self-paced exercise in COPD: a comparison of three tests. Chest. 2004; 126(3):766-73.

7. Cooper $\mathrm{KH}$. A means of assessing maximal oxygen intake. Correlation between field and treadmill testing. JAMA. 1968; 15; 203(3):201-4.

8. Enright PL. The six-minute walk test. Respir Care. 2003; 48(8):783-5.

9. Guyatt $\mathrm{GH}$, Pugsley SO, Sullivan MJ, Thompson PJ, Berman L, Jones NL, Fallen EL, Taylor DW. Effect of encouragement on walking test performance. Thorax. 1984; 39(11):818-22.

10. ATS Committee on Proficiency Standards for Clinical Pulmonary Function Laboratories. ATS statement: guidelines for the six-minute walk test. Am J Respir Crit Care Med 2002; 166(1):111-7.

11. Singh SJ, Morgan MD, Scott S, Walters D, Hardman AE. Development of a shuttle walking test of disability in patients with chronic airways obstruction. Thorax. 1992; 47(12):1019-24.

12. da Cunha-Filho IT, Pereira DA, de Carvalho AM, Campedeli L, Soares M, de Sousa FJ. The reliability of walking tests in people with claudication. Am J Phys Med Rehabil. 2007; 86(7):574-82.

13. Zainuldin R, Mackey MG, Alison JA. Prescription of walking exercise intensity from the incremental shuttle walk test in people with chronic obstructive pulmonary disease. Am J Phys Med Rehabil. 2012;91(7):592-600

14. Coelho CC, Aquino ES, de Almeida DC, Oliveira GC, Pinto RC, Rezende IM, Passos C. Comparative analysis and reproducibility of the modified shuttle walk test in normal children and in children with cystic fibrosis. J Bras Pneumol. 2007;33(2):168-74.

15. Bradley J, Howard J, Wallace E, Elborn S. Validity of a modified shuttle test in adult cystic fibrosis. Thorax. 1999; 54(5):437-9.

16. Selvadurai HC, Cooper PJ, Meyers N, Blimkie CJ, Smith L, Mellis CM, Van Asperen PP. Validation of shuttle tests in children with cystic fibrosis. Pediatr Pulmonol. 2003; 35(2):133-8.

17. Vagaggini $B$, Taccola M, Severino S, Marcello M, Antonelli S, Brogi S, De Simone C, Giardina A, Paggiaro PL. Shuttle walking test and 6-minute walking test induce a similar cardiorespiratory performance in patients recovering from an acute exacerbation of chronic obstructive pulmonary disease. Respiration. 2003; 70(6):579-84.

18. Rosa FW, Camelier A, Mayer A, Jardim JR. Evaluating 
physical capacity in patients with chronic obstructive pulmonary disease: comparing the shuttle walk test with the encouraged 6-minute walk test. J Bras Pneumol. 2006; 32(2):106-13.

19. Campo LA, Chilingaryan G, Berg K, Paradis B, Mazer B. Validity and reliability of the modified shuttle walk test in patients with chronic obstructive pulmonary disease. Arch Phys Med Rehabil. 2006; 87(7):918-22.

20. Dyer CA, Singh SJ, Stockley RA, Sinclair AJ, Hill SL. The incremental shuttle walking test in elderly people with chronic airflow limitation. Thorax. 2002; 57(1):34-8.

21. Green DJ, Watts K, Rankin S, Wong P, O'Driscoll JG. A comparison of the shuttle and 6 minute walking tests with measured peak oxygen consumption in patients with hear failure. J Sci Med Sport. 2001; 4(3):292-300.

22. Zwierska I, Nawaz S, Walker RD, Wood RF, Pockley AG, Saxton JM. Treadmill versus shuttle walk tests of walking ability in intermittent claudication. Med Sci Sports Exerc. 2004; 36(11):1835-40.

23. Moloney ED, Clayton N, Mukherjee DK, Gallagher CG, Egan JJ. The shuttle walk exercise test in idiopathic pulmonary fibrosis. Respir Med. 2003;97(6):682-7.

24. Seixas DM, Seixas DM, Pereira MC, Moreira MM, Paschoal IA. Oxygen desaturation in healthy subjects undergoing the incremental shuttle walk test. J Bras Pneumol. 2013; 39(4):440-6

25. Sívori M, Sáenz C. Shuttle in severe COPD patients Medicina (B Aires). 2010; 70(4):305-10.

26. Win T, Jackson A, Groves AM, Sharples LD, Charman SC Laroche CM. Comparison of shuttle walk with measured peak oxygen consumption in patients with operable lung cancer. Thorax. 2006; 61(1):57-60.

27. Payne GE, Skehan JD. Shuttle walking test: a new approach for evaluating patients with pacemakers. Heart. 1996;75(4):414-8.

28. Leger LA, Lambert J. A maximal multistage 20-m shuttle run test to predict VO2 max. Eur J Appl Physiol Occup Physiol.1982; 49(1):1-12.

29. Thompson WR, Gordon NF, Pescatello LS. Diretrizes do ACSM para testes de esforço e sua pescrição 2010. Rio de Janeiro: Ganabara Koogan; 2010.

30. Singh SJ, Jones PW, Evans R, Morgan MD. Minimum clinically important improvement for the incremental shuttle walking test. Thorax. 2008; 63(9):775-7.

31. Pepin V, Brodeur J, Lacasse Y, Milot J, Leblanc P, Whittom F, Maltais F. Six-minute walking versus shuttle walking: responsiveness to bronchodilation in chronic obstructive pulmonary disease. Thorax. 2007; 62(4):291-8.

32. Morales FJ, Montemayor T, Martinez A. Shuttle versus sixminute walk test in the prediction of outcome in chronic heart failure. Int J Cardiol. 2000;76(2-3):101-5.
33. Jolly K, Taylor RS, Lip GY, Singh S. Reproducibility and safety of the incremental shuttle walking test for cardiac rehabilitation. Int J Cardiol. 2008 28; 125(1):144-5.

34. Singh SJ, Morgan MD, Hardman AE, Rowe C, Bardsley PA. Comparison of oxygen uptake during a conventional treadmill test and the shuttle walking test in chronic airflow limitation. Eur Respir J 1994; 7(11):2016-20.

35. Solway S, Brooks D, Lacasse Y, Thomas S. A qualitative systematic overview of the measurement properties of functional walk tests used in the cardiorespiratory domain. Chest 2001; 119(1):256-70.

36. Morales FJ, Martinez A, Mendez M, Agarrado A, Ortega F, Fernandez-Guerra J, Montemayor T, Burgos J. A shuttle walk test for assessment of functional capacity in chronic heart failure. Am Heart J. 1999; 138(2 Pt 1):291-8.

37. Eiser N, Willsher D, Dore CJ. Reliability, repeatability and sensitivity to change of externally and self-paced walking tests in COPD patients. Respir Med. 2003;97(4):407-14

38. Pepera G, McAllister J, Sandercock G. Long-term reliability of the incremental shuttle walking test in clinically stable cardiovascular disease patients. Physiotherapy. 2010;96(3):222-7.

39. Fowler A, Singh SJ, Revill S. Reproducibility and validity of the incremental shuttle walking test in patients following coronary artery bypass surgery. Physiotherapy. 2005; 91(1):22-7.

40. Arnott AS. Assessment of functional capacity in cardiac rehabilitation. Coronary Health Care.1997;1:30-6.

41. Jurgensen SP, Antunes LC, Tanni SE, Banov MC, Lucheta PA, Bucceroni AF, Godoy I, Dourado VZ. The incremental shuttle walk test in older Brazilian adults. Respiration. 2011; 81(3):223-8.

42. Dourado VZ, Vidotto MC, Guerra RL. Reference equations for the performance of healthy adults on field walking tests. J Bras Pneumol. 2011; 37(5):607-14.

43. Dourado VZ, Guerra RL, Tanni SE, Antunes LC, Godoy I. Reference values for the incremental shuttle walk test in healthy subjects: from the walk distance to physiological responses. J Bras Pneumol. 2013; 39(2):190-7.

44. Probst VS, Hernandes NA, Teixeira DC, Felcar JM, Mesquita RB, Gonçalves CG, Hayashi D, Singh S, Pitta F. Reference values for the incremental shuttle walking test. Respir Med. 2012; 106(2):243-8.

\section{Endereço para correspondência:}

Danielle Aparecida Gomes Pereira Av. Antônio Carlos $n^{\circ} 6627$

Belo Horizonte/MG - CEP 31270-091

Telefone: +553134094783

E-mail: danielleufmg@gmail.com 